\title{
CFD Analysis of Heat Transfer in Ventilated Attics
}

\author{
Vijay Shankar1, Andreas Bengtson², Victor Fransson², Carl-Eric Hagentoft ${ }^{2}$ \\ ${ }^{1}$ Luleå University of Technology \\ Luleå, Sweden \\ vijay.shankar@ltu.se \\ ${ }^{2}$ Chalmers University of Technology \\ Gothenburg, Sweden
}

\begin{abstract}
Due to improper design of cold ventilated attics with regard to family homes, mild growth has become an increasing problem in Scandinavia and other cold countries around the globe. In this research paper, the influence of combined natural and forced convection on the thermal properties of insulation is investigated. The governing equations for fluid motion, energy (heat) and suitable turbulence model have been solved with help virtual numerical technique namely CFD. The numerical computations are conducted for two different insulations with varying values of permeability. The results of this complicated and sensitive heat transfer process are presented as function of different inlet velocities, physical properties of insulation and temperature difference across the calculation domain for full scale cold ventilated attics.
\end{abstract}

Keywords: Computational fluid dynamics (CFD), Heat transfer, Building physics, Fluid mechanics

\section{Introduction}

This article is a continuation of the research work performed by the authors in a previous article [1]. The previous article treated the impact of natural convection heat transfer in a principal cold attic model with wooden joists and insulation. In this article, the same geometry was used in order to investigate the effect of forced convection in the air cavity above the insulation. As in the previous work, ANSYS Fluent was used for the simulations, along with the same mesh as in the previous article. Forced convection is more or less always present in cold attics. This is due to the ventilation system of the attic, which consists of gaps on the sides of the attic where cold air flows in over the wind deflectors. Air can then flow out from the attic through the top of the roof. The ventilation system and the cross-sectional geometry of the attic can be seen in fig. 1. Simulations were performed with a constant temperature at the bottom of the attic, $\mathrm{T}_{\text {hot }}$, of $294 \mathrm{~K}$, and varying, lower, temperatures at the boundary of the roof of the same, $\mathrm{T}_{\text {cold. }}$. The temperature difference was varied between 10 to $50 \mathrm{~K}$.

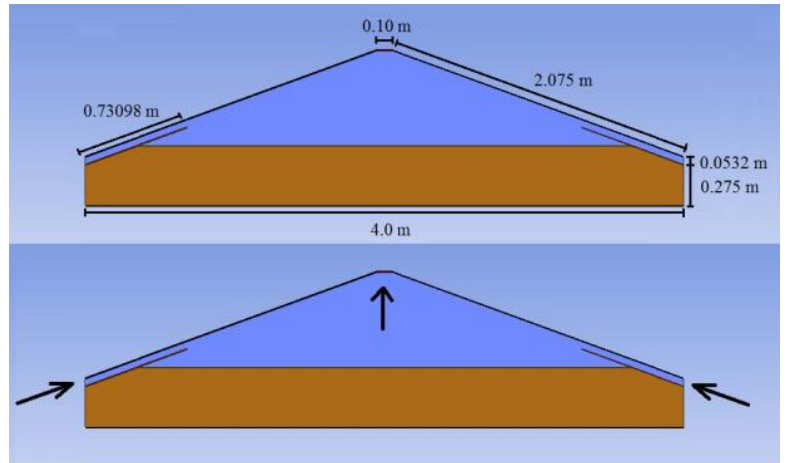

Fig. 1: The attic model, seen from the side. The upper region represents the air cavity and the lower one the porous insulation. The upper picture shows the dimensions of the principle attic model, and the lower picture visually represents the inlet and outlet of the ventilation system. 
combined with an air cavity. This model was developed by the authors and presented in an earlier article [2]. This model is in turn based on previous research [3-10], and verified by comparison to experimental work performed by Serkitjis (1995) [11].

\section{Numerical Setup}

The same numerical setup as in the previous work [1] and [2] was used for the simulations presented in this article. The boundary conditions applied for the attic model were mostly identical to the boundary conditions used in the investigation of natural convection in the attic [2]. These include specified constant temperatures of $\mathrm{T}=\mathrm{T}_{\text {cold }}$ on the roof of the attic, and $\mathrm{T}=\mathrm{T}_{\text {hot }}$ on the floor of the attic.
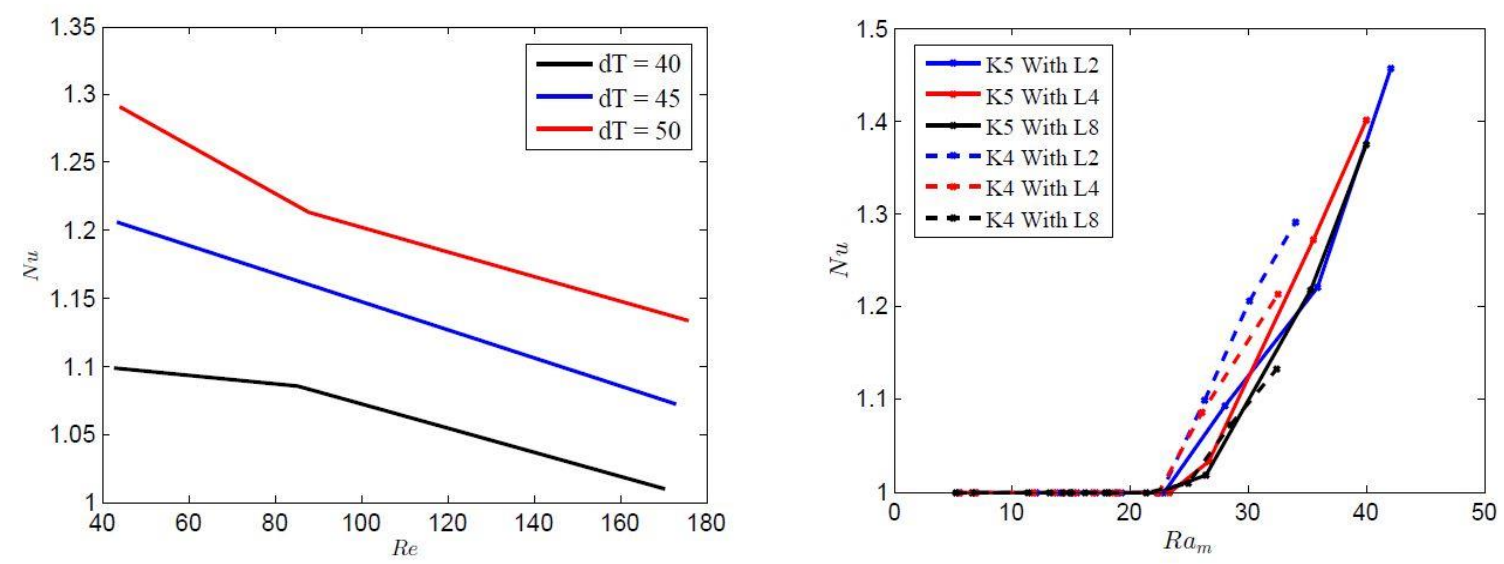

Fig. 2: (a) Plot of The Nusselt number versus the modified Rayleigh number for the cases with permeability of $4 * 10^{-8} \mathrm{~m}^{2}$ (the three dashed lines) and the cases with permeability of $5 * 10^{-8} \mathrm{~m}^{2}$ (the three solid lines). L2, L4 and L8 represents two, four and eight complete air displacements per hour, respectively. (b) Plot of The Nusselt number versus the Reynolds number for the cases with permeability of $4 * 10^{-8} \mathrm{~m}^{2}$. The Reynolds number are based on the geometry of the inlet. Each line represents one temperature difference.

The vertical boundaries in contact with the insulation were set to have a heat flux of $q=0$, and a symmetry boundary condition was specified for the sides of the attic parallel to the joists longitudinal direction. The differences in boundary conditions between the case with only natural convection Shankar, Bengtson and Fransson (2014) and the cases including forced convection was the modeling of the ventilation system. The boundaries situated between the wind deflectors and the roof were set to velocity inlets, with the inlet velocity set to correspond to the particular rate of complete air displacement of the simulation. The temperature of the inlet air was set to $\mathrm{T}=\mathrm{T}_{\text {cold. }}$ A pressure outlet boundary condition with atmospheric pressure was specified for the boundary at the very top of the roof. In fig. 1, the exact position of these boundaries can be seen.

\section{Results}

The Nusselt number was computed in the same way as in the previous work Shankar, Bengtson and Fransson (2014) and Shankar, Bengtson and Fransson (2014). For each rate of air exchange, nine temperature differences were computed. Since two different permeabilities were investigated, the total number of simulations for this work was 54 . The scale in each temperature contour picture goes from $294 \mathrm{~K}$ to $294 \mathrm{~K}$ minus the temperature difference, with red being the warmest These Two different types of insulation with different values of permeability were investigated, as well as three different inlet velocities for the ventilation. The three velocities correspond to three different rates of complete air displacement in the attic

are two, four and eight complete displacements per hour, respectively. The same methodology as in the previous article Shankar, Bengtson and Fransson (2014) was used for simulating the heat transfer in a medium containing porous media temperatures (bottom surface) and blue being the coldest (top surface). The temperature contour plots can be seen in fig. 4. The velocity vectors can be seen in fig. $5 \mathrm{fig}$. 6 . The exact velocities in these pictures are not of large interest, A plane parallel to the joists longitudinal direction in the middle of the attic was used to display the results in fig. 4 , and fig. 
5, whereas a plane perpendicular to the joists longitudinal direction in the middle of the attic was used to display the results in fig. 6 and fig. 7.

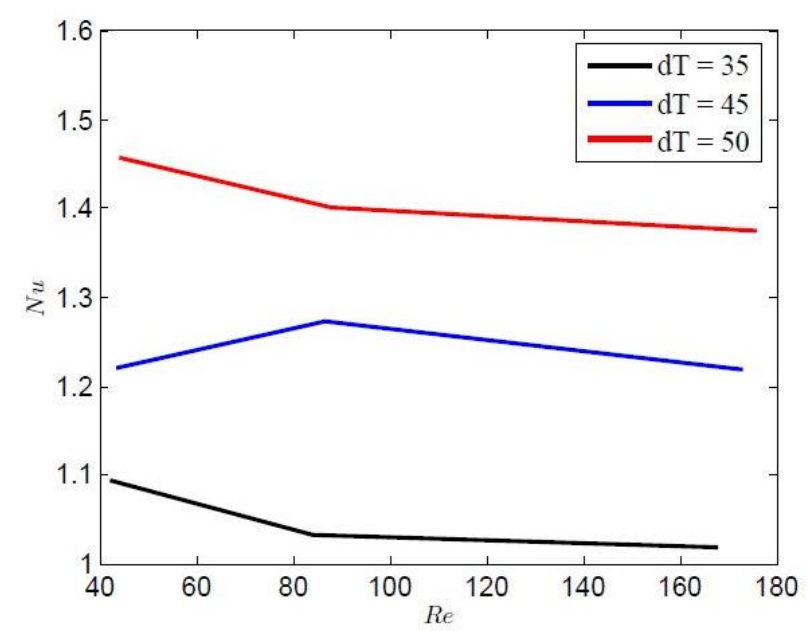

Fig. 3: Plot of The Nusselt number versus the Reynolds number for the cases with permeability of $5 * 10^{-8} \mathrm{~m}^{2}$. The Reynolds number are based on the geometry of the inlet. Each line represents one temperature difference.
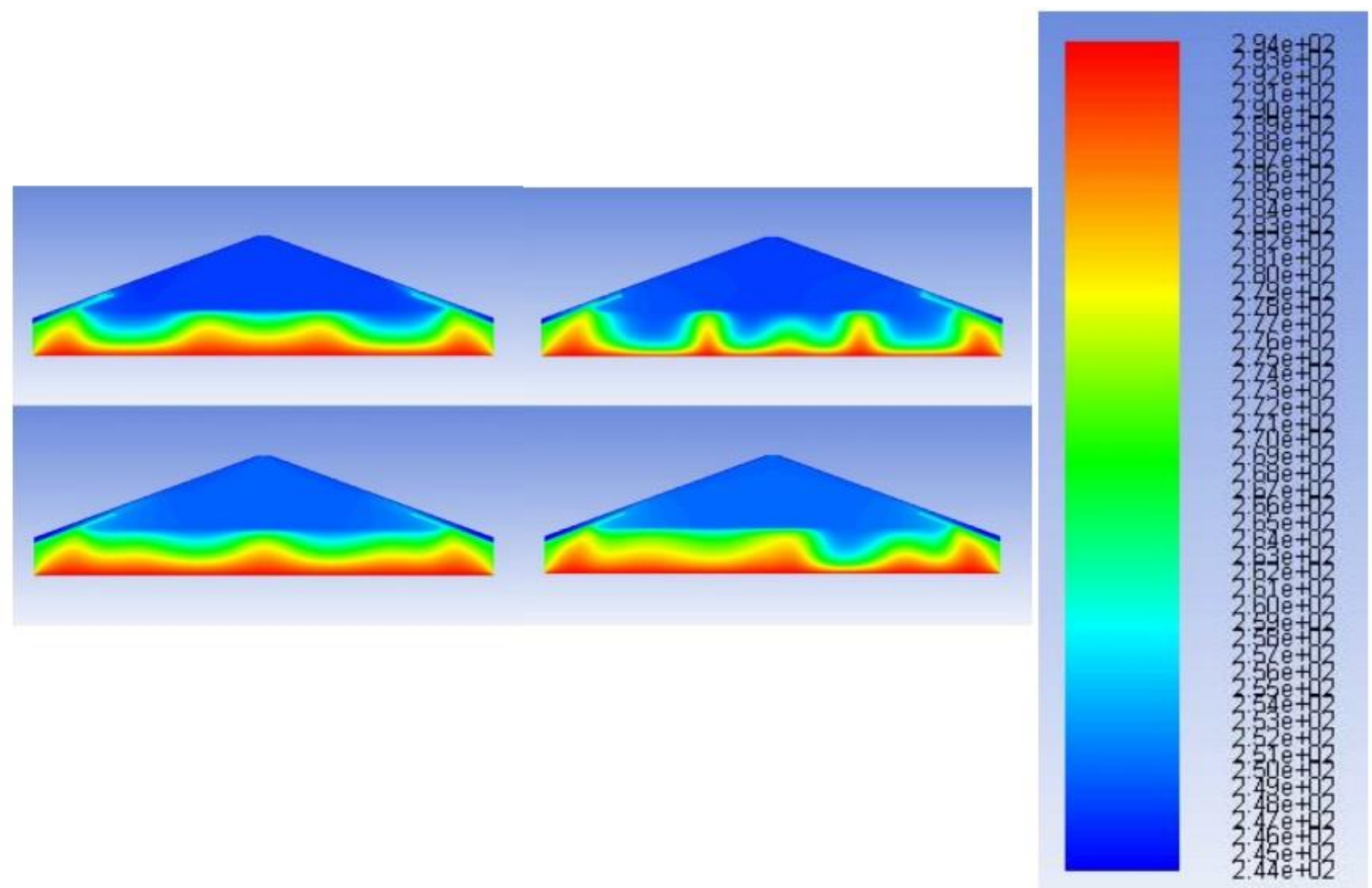

Fig. 4: (a) Temperature contours for the temperature difference of $35 \mathrm{~K}$ and permeability of $4 * 10^{-8} \mathrm{~m}^{2}$ visualized in a plane parallel to the joists located in the middle of the domain. The pictures are for, in order from above, the case with two respectively eight complete air displacements per hour. (b) Temperature contours for the temperature difference of $50 \mathrm{~K}$ and permeability of $4 * 10^{-8} \mathrm{~m}^{2}$ visualized in a plane parallel to the joists located in the middle of the domain. The pictures are for, in order from above, the case with two respectively eight complete air displacements per hour. 

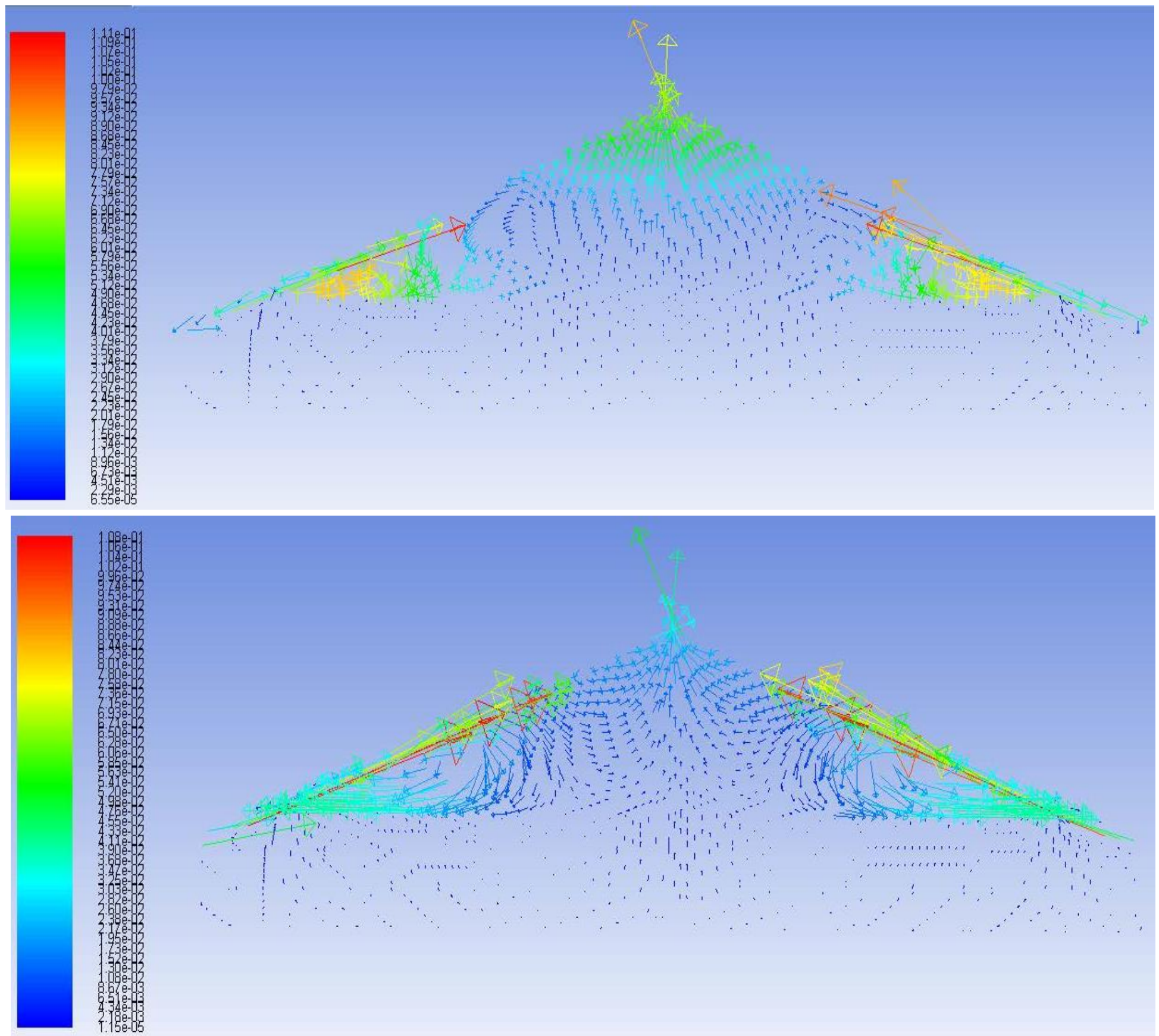

Fig. 5: (a)Velocity vectors for the temperature difference of $35 \mathrm{~K}$ and permeability of $4 * 10^{-8} \mathrm{~m}^{2}$ visualized in a plane parallel to the joists located in the middle of the domain. The pictures are for, in order from above, the case with two respectively eight complete air displacements per hour.

\section{Discussion}

As can be seen in fig. 2(a), the different air displacement rates generally has a small effect on the Nusselt versus the modified Rayleigh number curves. The shape of the curves is also very similar to those representing the case of pure natural convection in the previous article Shankar, Bengtson and Fransson (2014). The onset of natural convection occurs, just like in the simulations involving pure natural convection, approximately at $\mathrm{Ra}_{\mathrm{m}}=22$. The different air displacement rates seem to not affect the onset of natural convection at all. 

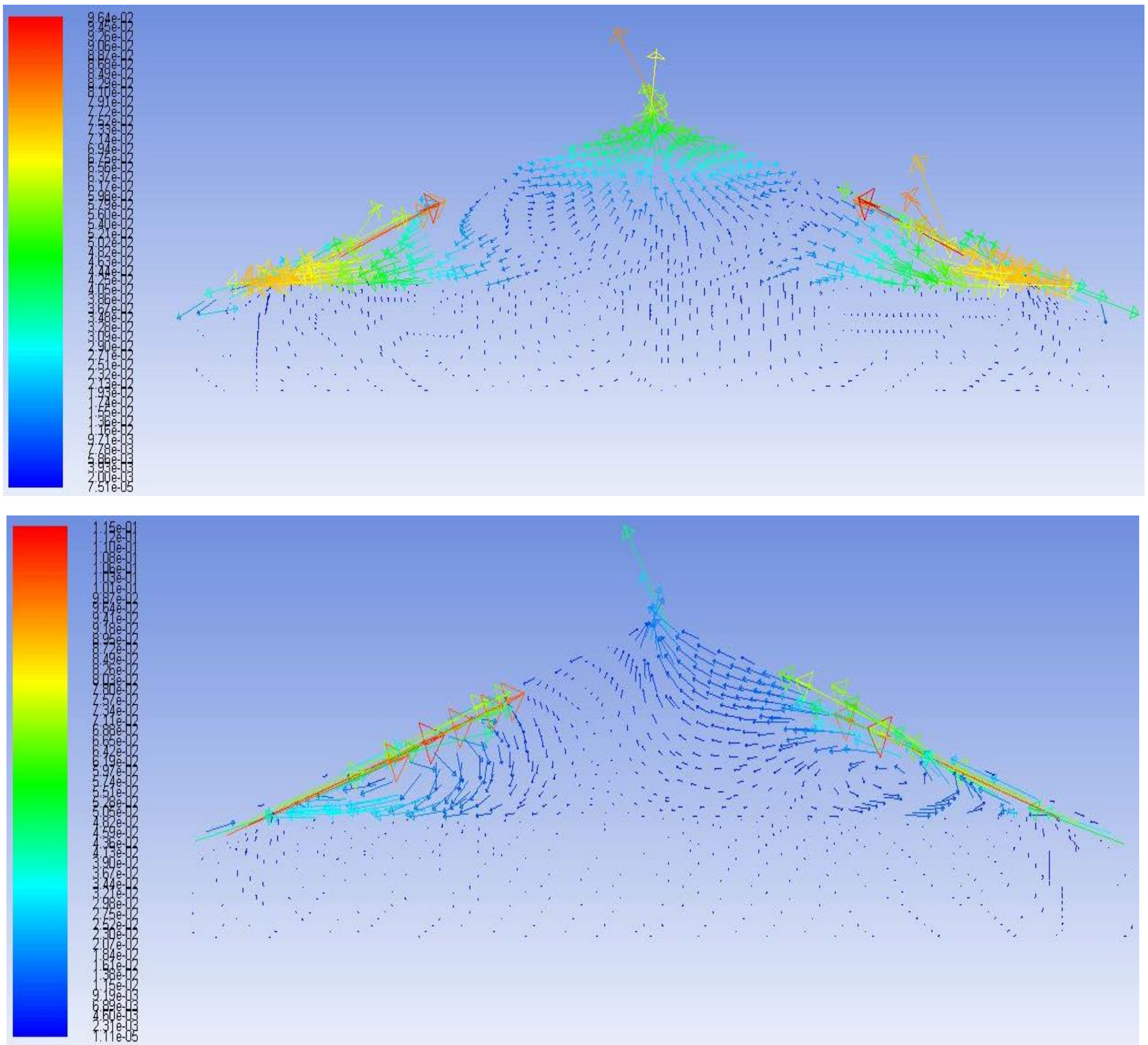

Fig. 5: (b) Velocity vectors for the temperature difference of $35 \mathrm{~K}$ and permeability of $5 * 10^{-8} \mathrm{~m}^{2}$ visualized in a plane parallel to the joists located in the middle of the domain. The pictures are for, in order from above, the case with two respectively eight complete air displacements per hour. 

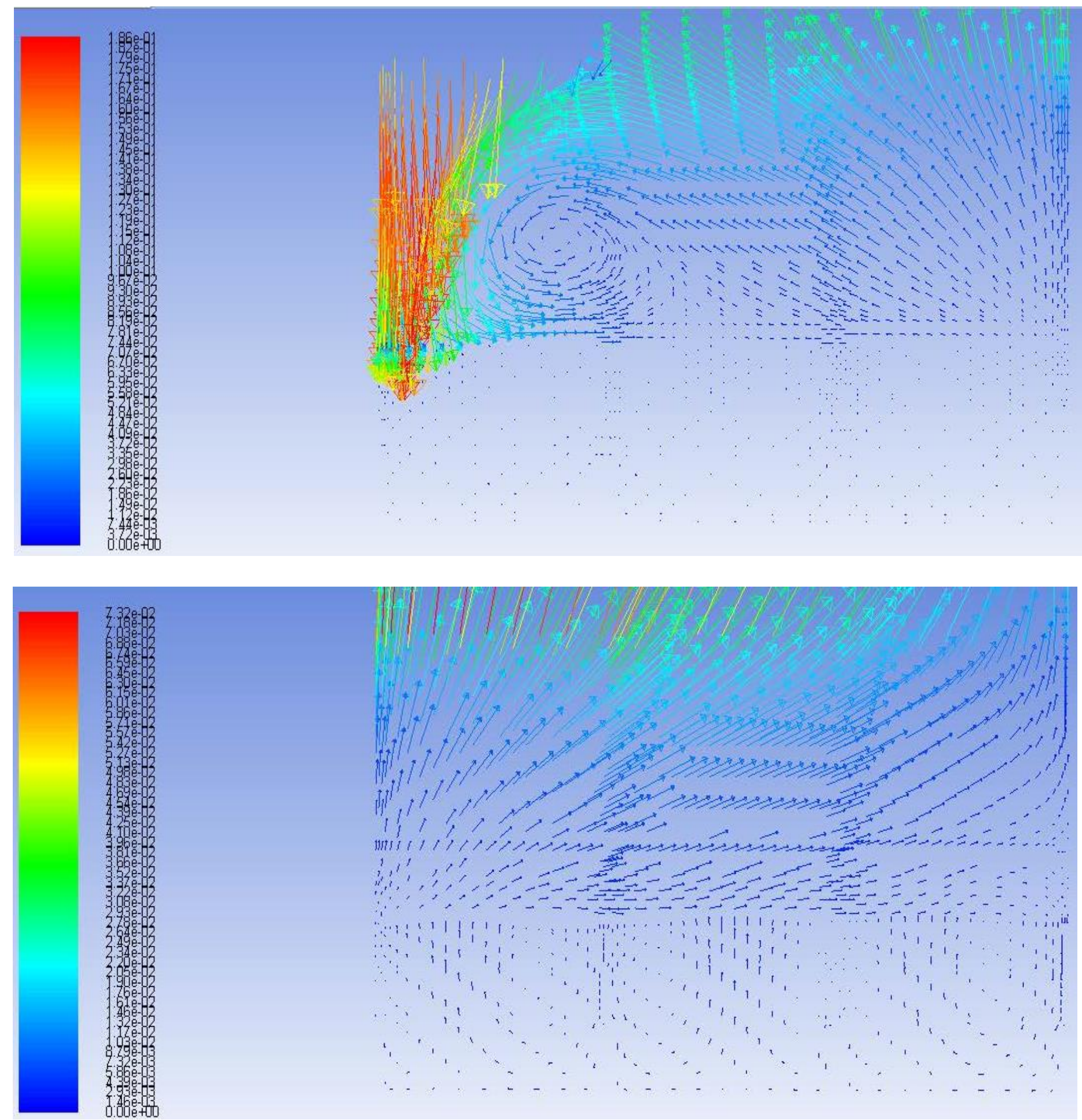

Fig. 6: (a)Velocity vectors for the temperature difference of $35 \mathrm{~K}$ and permeability of $4 * 10^{-8} \mathrm{~m}^{2}$ visualized in a plane perpendicular to the joists located in the middle of the domain. The plots are for, in order from above, the case with two respectively eight complete air displacements per hour. 

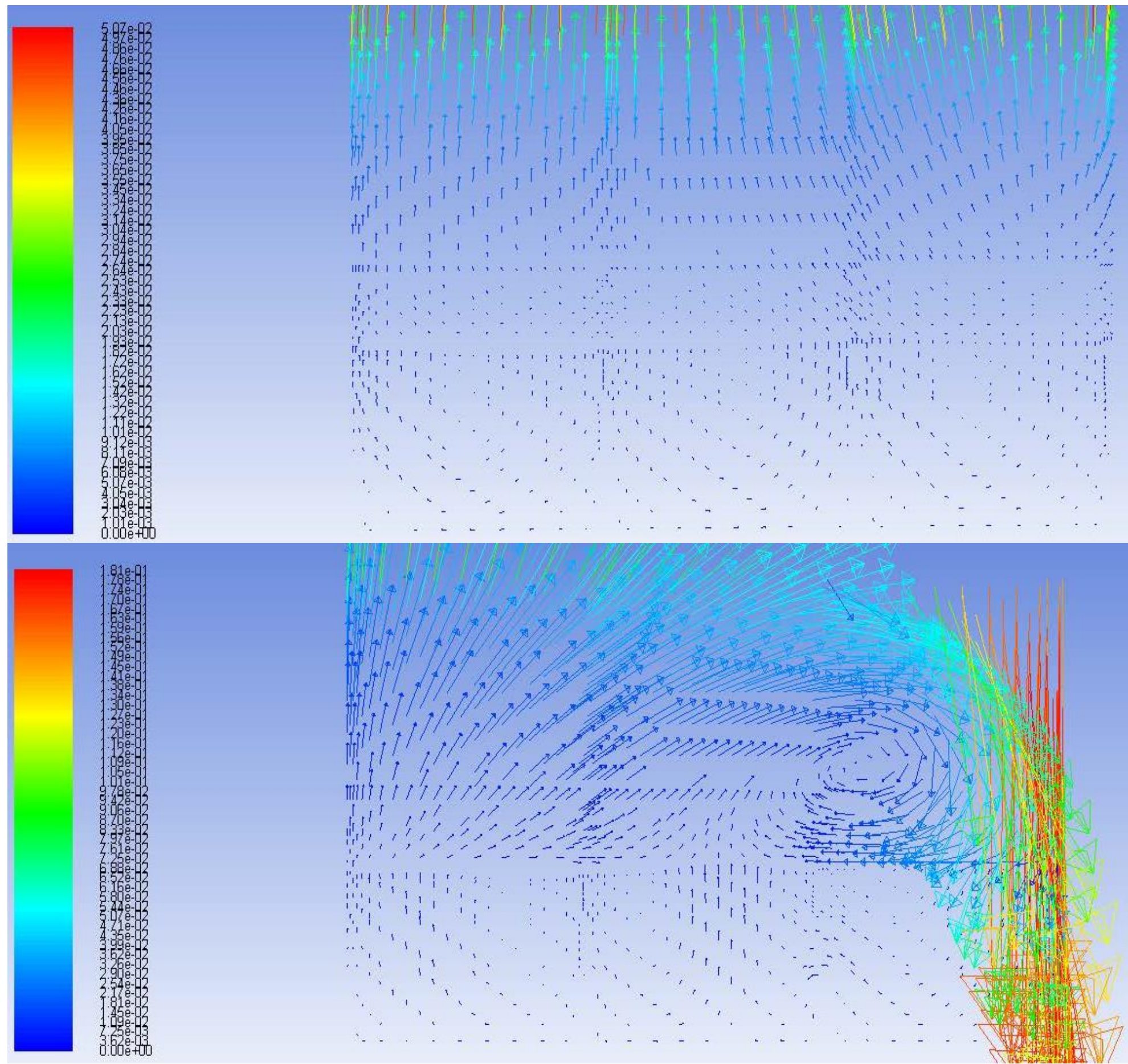

Fig. 6: (b) Velocity vectors for the temperature difference of $35 \mathrm{~K}$ and permeability of $5 * 10^{-8} \mathrm{~m}^{2}$ visualized in a plane perpendicular to the joists located in the middle of the domain. The plots are for, in order from above, the case with two respectively eight complete air displacements per hour.

Although the curves look very similar in fig. 2(a), there are some distinctions which can be seen clearer when studying the Nusselt number versus the Reynolds number in fig. 2(b) and fig. 3. These pictures show that as the Reynolds number, i.e. the inlet velocity, increases, the Nusselt number generally decreases. This decrease is, for some reason, more distinct for the simulations performed with the lower permeability of $4108 \mathrm{~m}^{2}$. Fig. 4 shows how the temperature field changes as the ventilation rate goes from two to eight air displacements per hour for the temperature differences of 35 and $50 \mathrm{~K}$ and the 
permeability of $4 * 10^{-8} \mathrm{~m}^{2}$. It is hard to see any tendencies in these figures as the ventilation rate becomes higher, all the pictures look very unsymmetrical. In the previous work with only natural convection on the attic model, the temperature contour plots were also very unsymmetrical and irregular, and showed no real trends as the temperature increased Shankar, Bengtson and Fransson (2014). However, in the results from the simulations on the box model, Shankar, Bengtson and Fransson (2014), there were clear trends in the figures as the temperature increased. Also, essentially all these simulations showed symmetrical results for the temperature contours. Based on this, it seems like the simulations for the attic model, both with and without ventilation enabled, were a lot more unsteady than in the case of the box model. The difference in the flow field due to the different air flow rates can be viewed for the temperature difference of $35 \mathrm{~K}$ in fig. 5 , fig. 6 . These pictures show the difference in the flow field when the air displacement rates are two respectively eight complete displacements per hour.

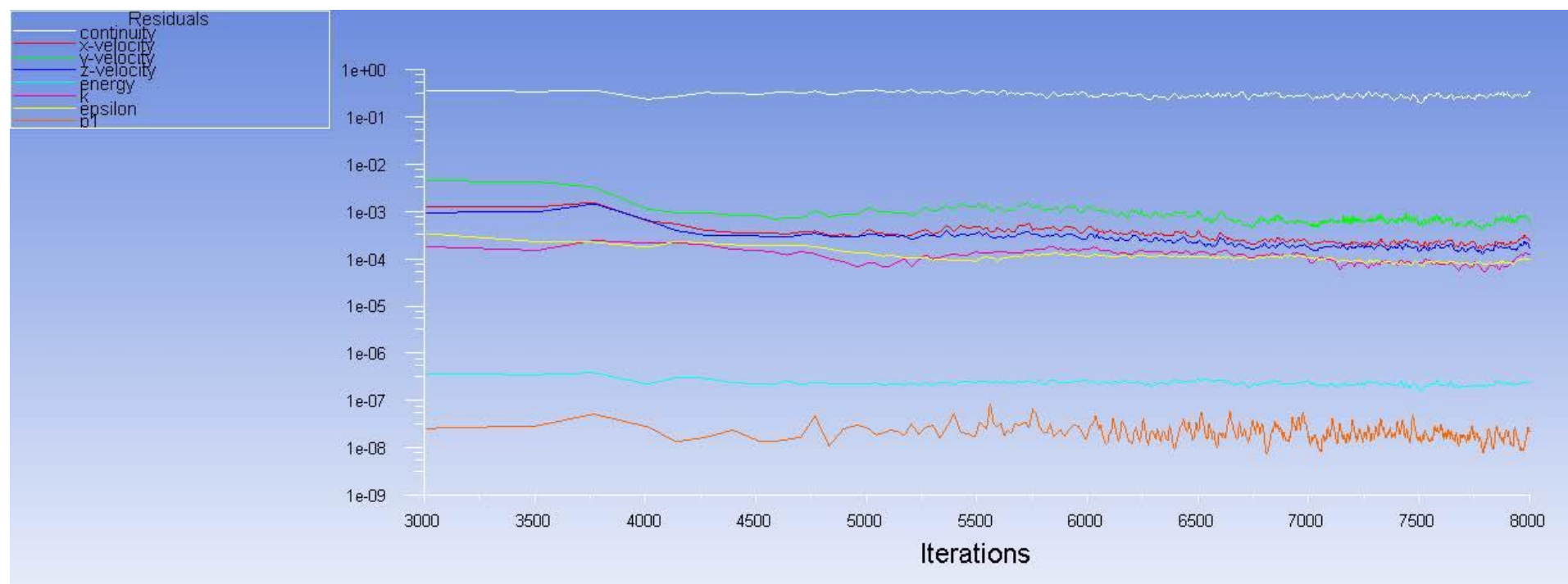

Fig. 7: Computational residuals for a simulation with temperature difference of $35 \mathrm{~K}$, permeability of $5 * 10^{-8} \mathrm{~m}^{2}$ and two complete air displacements per hour.

The flow field pictures taken in a plane parallel to the joists, fig. 5 shows that the same flow pattern occurs for both permeabilities. The inlet air forces the air in the attic to flow towards the middle of the air cavity of the attic and further out through the outlet on the top of the roof, with increased velocity when the air displacement rate increases. The flow field pictures taken in a plane perpendicular to the joints, fig. 6 shows some interesting phenomena.

As seen in the upper picture in both those figures, swirls have been developed in the air cavity for the lower air displacement rate. When the ventilation rate increases to eight air displacements per hour both these swirls are gone. This is probably due to as the ventilation rate increases, the overall flow field in the attic becomes more structured which prevents the formation of swirls and other irregular flow phenomena. The accuracy of the simulations has been verified in two ways. Firstly, the mass flow in and out has been compared. These results showed that the net mass flow for all of the performed simulations was very close to zero, which implies that continuity is ensured. Secondly, the computational residuals of the simulations have been investigated. For the simulation case with a temperature difference of $35 \mathrm{~K}$, a complete air displacement rate of two times per hour, and permeability of $5 * 10^{-8} \mathrm{~m}^{2}$ the residuals are presented in fig. 7 . These residuals are representable for how the residuals looked like for the simulations in this research work. The figure shows that the residuals are relatively low in general and does not, except for the radiation equation residuals (P1), fluctuate very much. However, just as in the previous work with only natural convection on the attic model Shankar, Bengtson and Fransson (2014) the residuals for continuity are, with a value around $4 * 10^{-1}$, really high. The values for all other residuals are good and all of them passes the general convergence criterion by Fluent ANSYS, Inc. (2011). 


\section{Conclusion}

As in the previous work conducted by the authors Shankar, Bengtson and Fransson (2014) and Shankar, Bengtson and Fransson (2014), the results when applying this methodology of simulating heat transfer and fluid flow in a porous media combined with an air cavity show a physically reasonable behavior. With the addition of a ventilation system, the attic model has become even more realistic and physical. The accuracy of the simulations has been verified by studying the net mass flux into the domain, and also by examining the computational residuals for the simulations. Except from high residuals in continuity, the simulations seem accurate in a numerical way. The results of the simulations involving forced convection show that the heat transfer in the insulation is largely unaffected by the addition of a ventilation system for this specific attic geometry. The inlet flow mainly affects the fluid movements in the air cavity. A change of total air displacement rate also has very little impact on the insulation heat transfer.

\section{Future Work}

The methodology used for simulating the heat transfer and fluid flow in the principle cold attic model gives good and, as it appear, accurate results. Because of this, the methodology can be applied in simulations to further investigate common phenomena in ventilate cold attics. A first step could be to investigate the effects of moisture transport, both through single- and multiphase flow. Since the heat transfer process involving natural convection always is unsteady in reality, it would also be interesting to perform unsteady simulations on the attic model. This would, however, require very large computational resources, especially considering that the simulations must be performed for a quite large period of time. It could also be of interest to perform simulations on a computational mesh with more cells, particularly to see if that would make the high residuals for continuity to go down.

\section{Acknowledgements}

This research work has been conducted with the help of financial and computer resources obtained from Swedish Energy Agency, Luleå University of Technology and the Division of Building Technology, Chalmers University of Technology.

\section{References}

[1] V. Shankar, A. Bengtson and V. Fransson, "Influence of Heat Transfer Processes in Porous Media with Air Cavity A CFD Analysis," in Proceedings of the 8th International Conference on Computational and Experimental Methods in Multiphase and Complex Flow, Valencia, Spain, 2015.

[2] A. Delmas and K. Wilkes, Numerical analysis of heat transfer by conduction and natural convection in loose-fill fiber glass insulation effects of convection on thermal insulation.,ORNL/CON-338, 1992.

[3] A. Delmas and E. Arquis "Early initiation of natural convection in an opened porous layer due to the presence of solid conductive inclusions," Journal of Heat and Mass Transfer, vol. 117, 1995.

[4] V. Shankar and C. E. Hagentoft, Numerical convection in insulating porous medium. Indoor Air 99, Edinburgh, 1999.

[5] V. Shankar and C. E. Hagentoft, Influence of natural convection on the thermal properties of insulating porous medium with air cavity. Indoor air 99, Edinburgh, 1999.

[6] V. Shankar and C.E. Hagentoft, Numerical investigation of natural convection in horizontal porous media heated from below comparisons with experiments. ASME International, Pittsburgh, 2000.

[7] V. Shankar, L. Davidson and E. Olsson, "Numerical investigation of turbulent plumes in both ambient and stratified surroundings," Journal of INDOOR AIR, Denmark, 1995.

[8] V. Shankar, L. Davidson and E. Olsson, Ventilation by displacement: Calculation of flow in vertical plumes., ROOM VENT, Aalborg, Denmark, 1992.

[9] P. Wahlgren, "Convection in Loose-fill Attic Insulation," Ph.D. Thesis, Chalmers University of Technology, Gteborg, 2001.

[10] M. Serkitjis, "Natural convection heat transfer in a horizontal thermal insulation layer underlying an air layer," $\mathrm{Ph} . \mathrm{D}$. Thesis, Chalmers University of Technology, Gteborg,1995.

[11] ANSYS, Inc., ANSYS FLUENT Users Guide, ANSYS, Inc, Canonsburg, 2011. 